\title{
Surface Roughness Prediction and Parameter Selection for Grinding Process with Computer Numerical Control
}

\author{
Cheng-Jian Lin, ${ }^{1,2 *}$ Jyun-Yu Jhang, ${ }^{3}$ Shou-Zheng Huang, ${ }^{4}$ and Ming-Yi Tsai ${ }^{4}$ \\ ${ }^{1}$ Department of Computer Science and Information Engineering, National Chin-Yi University of Technology, \\ Taichung 411, Taiwan \\ ${ }^{2}$ College of Intelligence, National Taichung University of Science and Technology, Taichung 404, Taiwan \\ ${ }^{3}$ Institute of Electrical and Control Engineering, National Yang Ming Chiao Tung University, Hsinchu 300, Taiwan \\ ${ }^{4}$ Institute of Precision Manufacturing, National Chin-Yi University of Technology, Taichung 411, Taiwan
}

(Received December 29, 2020; accepted March 24, 2021)

Keywords: grinding assistance system, grinding process, surface roughness prediction, differential evolution, type-2 TSK fuzzy neural network

We propose a novel intelligent grinding assistance system (IGAS) for the grinding of silicon carbide ( $\mathrm{SiC}$ ) with computer numerical control (CNC). The proposed IGAS predicts surface roughness $(\mathrm{Ra})$ and suggests suitable parameters for the grinding process. To establish the Ra prediction model, a type-2 functional-link-based fuzzy neural network (T2FLFNN), which updates the network parameter by Lévy-based dynamic group differential evolution (LDGDE), is developed. The LDGDE includes the Lévy flight and dynamic group mechanism to improve the shortcomings of the traditional differential evolution (DE) algorithm. Subsequently, DE is adopted to optimize the grinding parameters according to user requirements. Experimental results of practical machining show that the mean absolute percentage error (MAPE) using the IGAS is as low as $1.62 \%$. Therefore, the proposed IGAS can provide suitable grinding parameters according to the requirements of users.

\section{Introduction}

Silicon carbide $(\mathrm{SiC})$ is a semiconductor material composed of silicon $(\mathrm{Si})$ and carbon $(\mathrm{C})$. $\mathrm{SiC}$ has a strong bonding force, is chemically and mechanically stable, and has been widely used in electronic parts, vehicles, and aerospace. ${ }^{(1-3)}$ However, SiC has high hardness and brittleness and poor workability. Therefore, the processing of $\mathrm{SiC}$ materials is challenging. Because $\mathrm{SiC}$ has low fracture toughness, attempts to machine it may result in severe edge fracture and deterioration of quality. Many researchers have used an ultrasound-assisted grinding system to machine SiC. Liu et al. ${ }^{(4)}$ discussed the tool wear problem in conventional milling and ultrasonicvibration-assisted milling. They found that the ultrasonic vibration milling used in machining can reduce tool wear. Increasing the ultrasonic amplitude also increases tool wear. Wang et al. ${ }^{(5)}$ carried out a single abrasive ultrasonic vibration cutting test. Compared with ordinary cutting, ultrasonic-vibration-assisted grinding reduced the grinding force by about $60 \%$ and achieved

*Corresponding author: e-mail: cjlin@ncut.edu.tw https://doi.org/10.18494/SAM.2021.3269 
high-efficiency processing of $\mathrm{SiC}$ composite materials with low damage. Chen et al. ${ }^{(6)}$ used a PCD tool for ultrasonic-assisted milling of SiC composites. They pointed out that ultrasonic vibration of an appropriate amplitude promotes the removal of carbon fibers in micro-brittle fracture and reduces the roughness of the processed surface. These studies indicate that ultrasound-assisted machining can enhance machining quality.

Different grinding parameters will result in different surface roughness (Ra). Predicting Ra for different combinations of parameters is an important issue. Multivariate analysis (such as regression analysis) has been widely used to build predictive models. Kong et al. ${ }^{(7)}$ designed an Ra prediction model by adopting Bayesian linear regression (BLR). They also analyzed the performance of different models including standard BLR, Gaussian BLR, standard SBLR, and Gaussian SBLR. Experimental results showed that standard SBLR had the best predictive performance among these models. Sudianto et al. ${ }^{(8)}$ employed several parameters such as feeding speed, spindle revolution, depth of cut, helix angle, length of cut, cutting speed, and nose radius of cut to predict Ra. The regression method was used to build the prediction model. The results showed that the proposed regression can effectively predict Ra from the machining parameters. However, the disadvantage of multivariate analysis is that it requires quite complex calculations to obtain satisfactory conclusions, and a large number of variables need to be collected from observations. Recently, artificial neural networks have been used to establish prediction models. Srikant et al. ${ }^{(9)}$ proposed a backpropagation neural network (BPNN) to build a tool wear prediction model. Experimental results showed that the BPNN model performed well in predicting tool wear with high accuracy. Cheri et al. ${ }^{(10)}$ designed a fuzzy neural network (FNN), which combined the concepts of fuzzy logic and neural networks, for surface roughness prediction of a cold-rolled steel strip. The fuzzy logic has a human reasoning mechanism and the neural network provides nonlinear approximation ability, which make the prediction performance of the FNN higher than that of a neural network. Although these methods can be applied successfully, the prediction model performance is unstable in a factory environment because noise introduces uncertainty into the input signal, affecting the prediction result. To resolve the problem of uncertainty, a type-2 fuzzy neural network (type-2 FNN) ${ }^{(11,12)}$ has been used to establish a prediction model. A type-2 FNN adopts fuzzy sets as membership values, which provide a footprint of the uncertainty to deal with the uncertainty problem. Lin et al. ${ }^{(13)}$ designed a type-2 FNN to solve prediction problems. Gaussian noise was added to the input signal to verify the type-2 FNN's anti-noise capability. The experimental results demonstrate that a type-2 FNN is more robust than an FNN. The aforementioned methods used the backpropagation (BP) algorithm ${ }^{(14,15)}$ to adjust the network parameters. Nevertheless, the BP algorithm has the disadvantage of falling into a local minimum.

To acquire the global optimum solution, many studies adopted evolutionary computation methods to optimize network parameters. The evolutionary algorithms include differential evolution $(\mathrm{DE}){ }_{(}^{(16,17)}$ particle swarm optimization (PSO), ${ }^{(18)}$ artificial bee colony $(\mathrm{ABC}){ }^{(19)}$ genetic algorithm (GA), ${ }^{(20)}$ and whale optimization algorithm (WOA). ${ }^{(21)}$ Compared with other evolutionary algorithms, DE has the advantages of rapid convergence, fewer parameters, and a simple structure. However, the traditional DE still easily falls into a local optimum when confronted with a complex problem. In this study, Lévy-based dynamic group differential 
evolution (LDGDE) is developed to enhance the traditional DE and improve the accuracy of the prediction model.

The above-mentioned methods only used the grinding processing parameters to predict Ra. That is, they did not consider using the Ra value to generate suitable grinding processing parameters for the user. The selected parameters of the grinding process will differ according to the quality requirements. Different combinations of grinding parameters corresponding to different qualities can be considered as an optimization problem. The effective provision of appropriate grinding parameters to users can not only save time but also improve processing efficiency. Therefore, we propose a novel intelligent grinding assistance system (IGAS) for grinding with computer numerical control (CNC) in this study. The IGAS adopted a type-2 functional-link-based fuzzy neural network (T2FLFNN) to construct the prediction network. The prediction accuracy of the designed T2FLFNN is compared with those of a BPNN and FNN. Then, PSO is utilized to optimize the grinding parameters. According to the requirements of the user, the proposed IGAS can suggest suitable parameters for the grinding process. Experimental results demonstrate the effectiveness of the proposed IGAS.

This paper is organized as follows. Section 2 introduces the experimental equipment. Section 3 outlines the proposed IGAS. The experimental results are discussed in Sect. 4. Section 5 presents the conclusion.

\section{Experimental Equipment}

\subsection{Material}

In this study, $\mathrm{SiC}$ is adopted as the material. The advantages of $\mathrm{SiC}$ are high thermal conductivity and excellent wear resistance. The properties of $\mathrm{SiC}$ are shown in Table 1.

\subsection{Ultrasonic tool holder and grinding pin}

A BT-40 ultrasonic tool holder (Hantop Intelligence Tech) is utilized to enhance the grinding quality. ${ }^{(2)}$ The specifications of the ultrasonic tool holder are shown in Fig. 1 and Table 2. In this study, a diamond grinding pin is used in the grinding process. The specifications of the diamond grinding pin are shown in Fig. 2 and Table 3.

Table 1

Mechanical properties of SiC.

\begin{tabular}{lcc}
\hline Mechanical & SI/Metric & Value \\
\hline Elastic modulus & $(\mathrm{GPa})$ & 410 \\
Compressive strength & $(\mathrm{MPa})$ & 3900 \\
Flexural strength & $(\mathrm{MPa})$ & 550 \\
Fracture toughness & $\left(\mathrm{MPa} \cdot \mathrm{ml} /{ }^{2}\right)$ & 4.6 \\
Density & $(\mathrm{gm} / \mathrm{cc})$ & 3.1 \\
Maximum use temperature & $\left({ }^{\circ} \mathrm{C}\right)$ & 1650 \\
Hardness & $\left(\mathrm{kg} / \mathrm{mm}^{2}\right)$ & 2800 \\
\hline
\end{tabular}



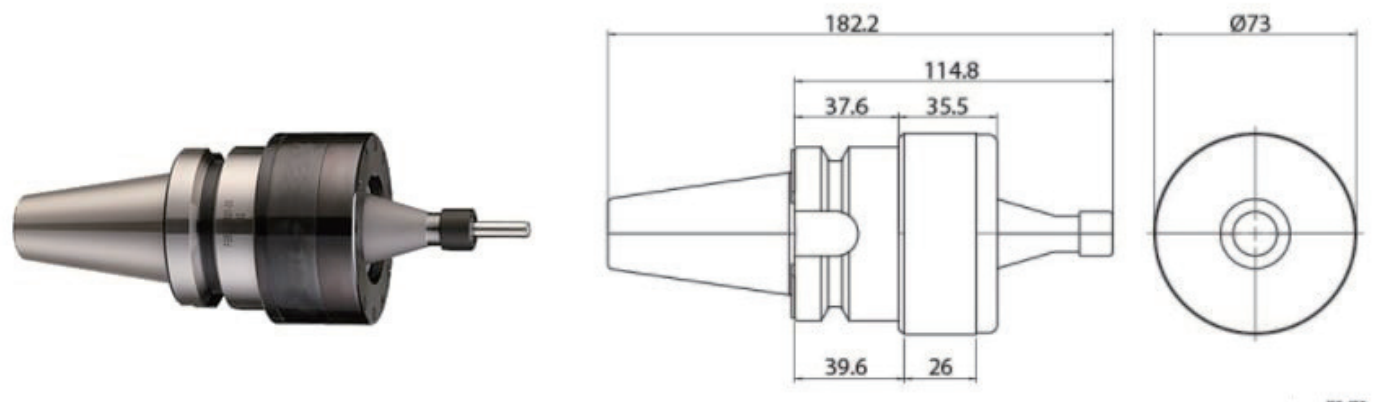

Fig. 1. (Color online) BT-40 ultrasonic tool holder.

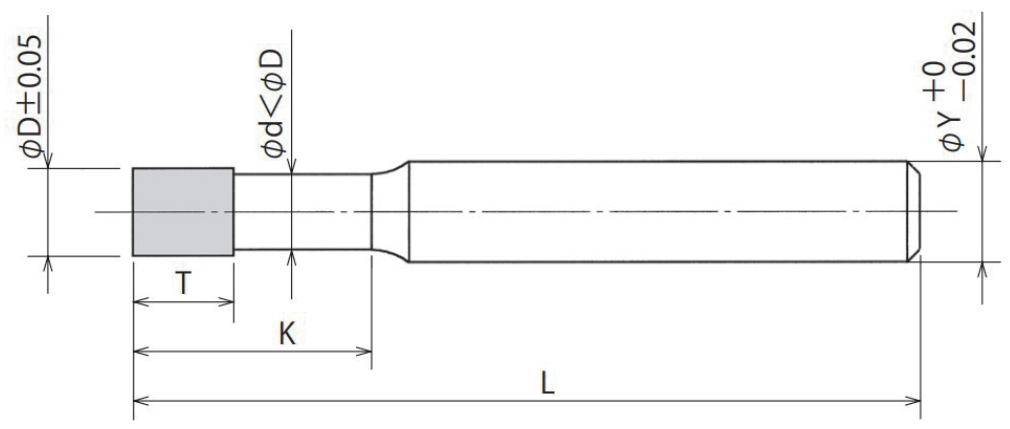

Fig. 2. Diamond grinding pin.

Table 2

Specifications of BT-40 ultrasonic tool holder.

\begin{tabular}{lc}
\hline Operating frequency & $20-32 \mathrm{kHz}$ \\
Weight & $2 \mathrm{~kg}$ \\
Maximum spindle speed & $24000 \mathrm{rpm}$ \\
Automatic tool changer & Yes \\
Runout & $<5 \mu \mathrm{m}$ \\
\hline
\end{tabular}

Table 3

Specifications of the diamond grinding pin.

\begin{tabular}{lc}
\hline Head (Ø D) & $6.0 \mathrm{~mm}$ \\
Width (T) & $5 \mathrm{~mm}$ \\
Collar (K) & $5 \mathrm{~mm}$ \\
Shank (Ø Y) & $8 \mathrm{~mm}$ \\
Length (L) & $80 \mathrm{~mm}$ \\
\hline
\end{tabular}

\subsection{CNC machine tool}

A QUASER-MV184C CNC machine tool ${ }^{(23)}$ is adopted to collect the grinding experimental data. The BT-40 tool holder is also installed in the CNC machine tool to reduce the processing time and improve the grinding quality. The QUASER-MV184C CNC machine tool contains a HEIDENHAIN TNC640 controller. Figure 3 and Table 4 present the machine tool and its specifications, respectively.

\subsection{Surface profiler}

The Ra of SiC was measured by a ZYGO NewView ${ }^{\mathrm{TM}} 8300$ 3D optical surface profiler ${ }^{(24)}$ after the grinding process. The profiler and its specifications are respectively shown in Fig. 4 and Table 5. 


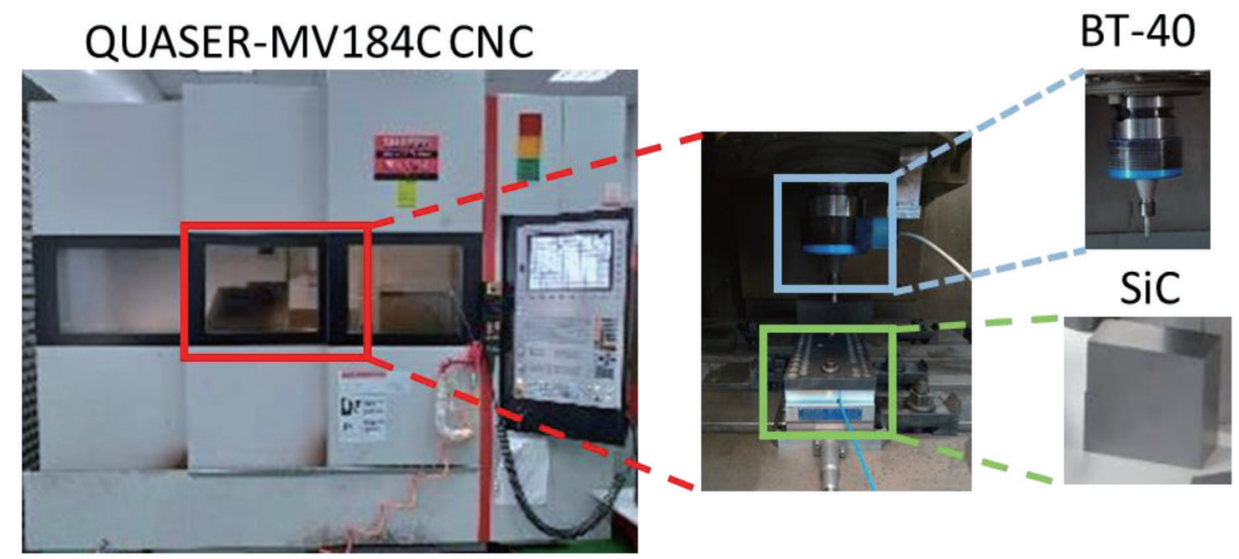

Fig. 3. (Color online) CNC machine tool with BT-40 tool holder.

Table 4

Specifications of the CNC machine tool.

\begin{tabular}{lcc}
\hline Table size & $(\mathrm{mm})$ & $1200 \times 600$ \\
Max spindle speed & $(\mathrm{rm})$ & 12000 \\
Travel X/Y/Z & $(\mathrm{mm})$ & $1020 / 610 / 610$ \\
Table load capacity & $(\mathrm{kg})$ & 500 \\
\hline
\end{tabular}

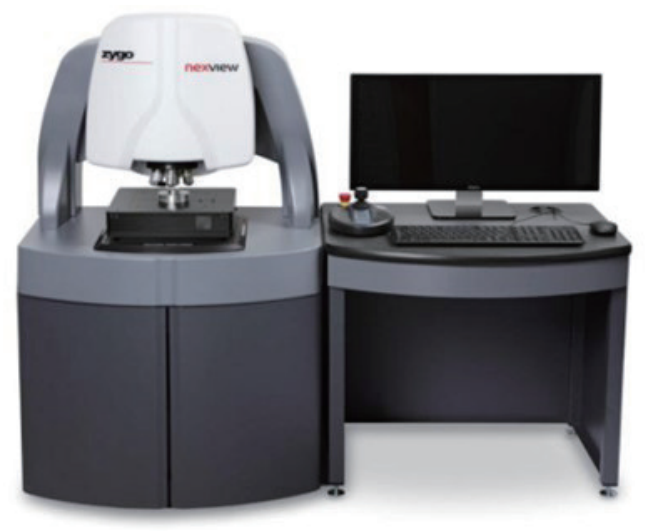

Fig. 4. (Color online) NewView ${ }^{\mathrm{TM}} 8300$ 3D optical surface profiler.

Table 5

Specifications of NewView ${ }^{\mathrm{TM}} 8300$.

\begin{tabular}{lc}
\hline Vertical scan range & $\begin{array}{c}20 \mathrm{~mm} \text { with extended scan } \\
\mu \mathrm{m} \text { with precision piezo drive }\end{array}$ \\
\hline Surface topography repeatability & $0.2 \mathrm{~nm}$ \\
\hline Repeatability of RMS & $0.01 \mathrm{~nm}$ \\
\hline Optical lateral resolution & $0.34 \mu \mathrm{m}(100 \times$ objective $)$ \\
\hline Spatial sampling & $0.04 \mu \mathrm{m}(100 \times$ objective $2 \times$ zoom $)$ \\
\hline Maximum data scan speed & $96 \mu \mathrm{m} / \mathrm{s}$ \\
\hline Height response linearity & $\leq 30 \mathrm{~nm}$ \\
\hline Step height repeatability & $0.1 \%$ \\
\hline Step height accuracy for extended scans & $0.8 \%$ \\
\hline
\end{tabular}




\section{Intelligent Grinding Assistance System (IGAS)}

In order to assist users in choosing suitable grinding parameters, the IGAS is introduced in this subsection. The IGAS contains two main functions: Ra prediction and grinding parameter optimization. To improve the accuracy of the prediction model, a T2FLFNN with an LDGDE parameter updating algorithm is developed to establish the prediction model. After that, PSO is adopted to optimize the grinding parameters.

\subsection{Proposed T2FLFNN for Ra prediction}

\subsubsection{T2FLFNN architecture}

The architecture of the T2FLFNN is presented in Fig. 5. The IF-THEN rule is defined as follows:

$$
\begin{aligned}
& \text { Rule }_{j} \text { : IF } \quad x_{1} \text { is } A_{1 j}, x_{2} \text { is } A_{2 j}, \ldots, \text { and } x_{i} \text { is } A_{i j}, \\
& \text { THEN } y_{j}=\sum_{k=1}^{M} \omega_{k j} \varphi_{k}=\omega_{1 j} \varphi_{1}+\omega_{2 j} \varphi_{2}+\ldots+\omega_{M j} \varphi_{M},
\end{aligned}
$$

where $x_{i}$ is the input, $y_{i}$ is the output, $A_{i j}$ represents the type- 2 fuzzy sets, $j=1,2, \ldots, R$ represents the rule number, $\omega_{k j}$ is the link weight, $M$ is the number of basis functions, and $\varphi_{k}$ represents the basis trigonometric function.

The Gaussian function in Eq. (2) is used as the type-2 fuzzy membership function, which has uncertainty mean $\left[m_{i j_{1}}, m_{i j_{2}}\right]$ and standard deviation $\sigma_{i j}$.

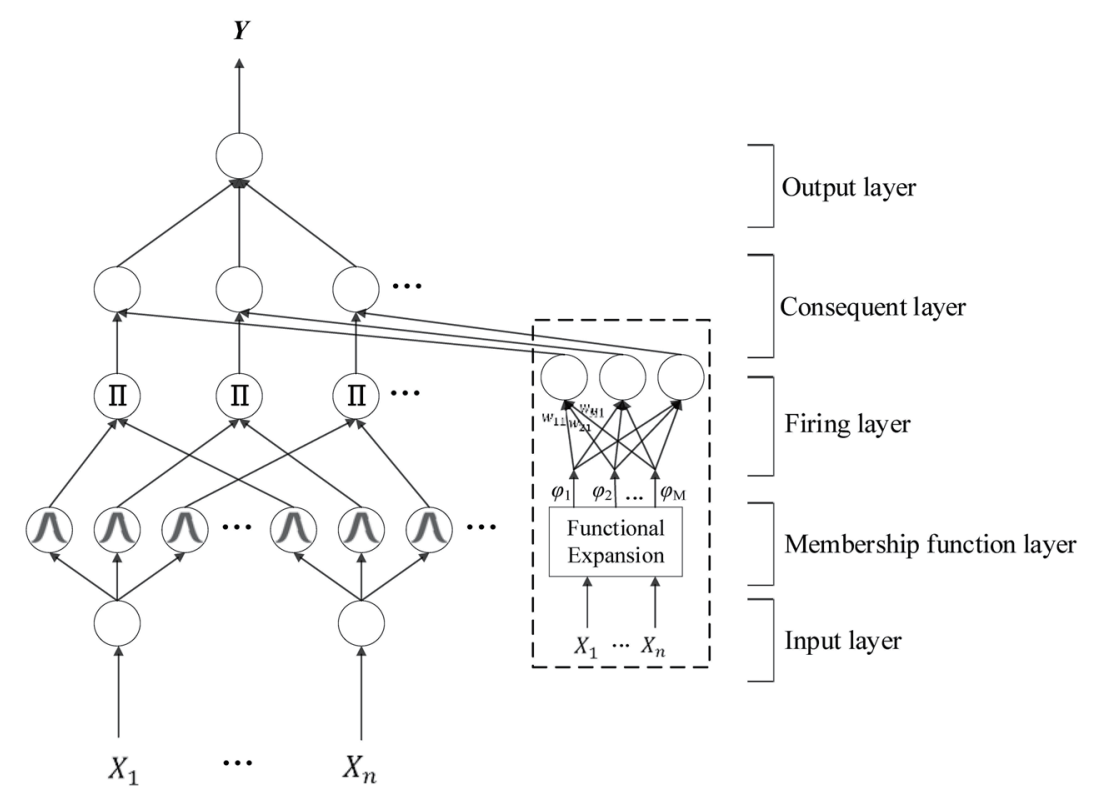

Fig. 5. Architecture of T2FLFNN. 


$$
u_{i j}^{(2)}=\exp \left(-\frac{\left[u_{i}^{(1)}-m_{i j}\right]^{2}}{\sigma_{i j}^{2}}\right) \equiv N\left(m_{i j}, \sigma_{i j} ; u_{i}^{(1)}\right), m_{i j} \in\left[m_{i j}^{(1)}, m_{i j}^{(2)}\right]
$$

The Gaussian membership function has an upper boundary $\bar{u}_{i j}^{(2)}$ and lower boundary $\underline{u}_{i j}^{(2)}$.

$$
\begin{gathered}
\bar{u}_{i j}^{(2)}= \begin{cases}N\left(m_{i j}^{(1)}, \sigma_{i j} ; u_{i}^{(1)}\right), & \text { if } u_{i}^{(1)}<m_{i j}^{(1)} \\
1, & \text { if } m_{i j}^{(1)} \leq u_{i}^{(1)} \leq m_{i j}^{(2)} \\
N\left(m_{i j}^{(2)}, \sigma_{i j} ; u_{i}^{(1)}\right), & \text { if } u_{i}^{(1)}>m_{i j}^{(2)}\end{cases} \\
\underline{u}_{i j}^{(2)}=\left\{\begin{array}{l}
N\left(m_{i j}^{(2)}, \sigma_{i j} ; u_{i}^{(1)}\right), \text { if } u_{i}^{(1)} \leq \frac{m_{i j}^{(1)}+m_{i j}^{(2)}}{2} \\
N\left(m_{i j}^{(1)}, \sigma_{i j} ; u_{i}^{(1)}\right), \text { if } u_{i}^{(1)}>\frac{m_{i j}^{(1)}+m_{i j}^{(2)}}{2}
\end{array}\right.
\end{gathered}
$$

The firing strengths $\bar{u}_{j}^{(3)}$ and $\underline{u}_{j}^{(3)}$ of each rule node are calculated by an algebraic product operation.

$$
\bar{u}_{j}^{(3)}=\prod_{i} \bar{u}_{i j}^{(2)} \text { and } \underline{u}_{j}^{(3)}=\prod_{i} \underline{u}_{i j}^{(2)}
$$

A method reducing the order is adopted to transform the type- 2 fuzzy set into a type- 1 fuzzy set.

$$
\bar{u}^{(4)}=\frac{\sum_{j=1}^{R} \bar{u}_{j}^{(3)}\left(\sum_{k=1}^{M} \omega_{k j} \varphi_{k}\right)}{\sum_{j=1}^{R} \bar{u}_{j}^{(3)}}, \underline{u}^{(4)}=\frac{\sum_{j=1}^{R} \underline{u}_{j}^{(3)}\left(\sum_{k=1}^{M} \omega_{k j} \varphi_{k}\right)}{\sum_{j=1}^{R} \underline{u}_{j}^{(3)}}
$$

Here, $\sum_{k=1}^{M} \omega_{k j} \varphi_{k}$ is a nonlinear combination of inputs. The functional expansion is based on a trigonometric function and defined as

$$
\left[\varphi_{1}, \varphi_{2}, \ldots, \varphi_{M}\right]=\left[x_{1}, \sin \left(\pi x_{1}\right), \cos \left(\pi x_{1}\right), \ldots, x_{n}, \sin \left(\pi x_{n}\right), \cos \left(\pi x_{n}\right)\right]
$$

The output is defuzzified using the average of $\bar{u}^{(4)}$ and $\underline{u}^{(4)}$. The output $y$ is obtained as

$$
y=\frac{\bar{u}^{(4)}+\underline{u}^{(4)}}{2} .
$$




\subsubsection{Parameter learning using LDGDE}

Traditional DE has many shortcomings such as trapping at local optima and low precision. Therefore, LDGDE with the Lévy flight and dynamic group mechanism is proposed to overcome the shortcomings of DE. The pseudocode of LDGDE is shown in Fig. 6.

The steps are expressed as follows:

Coding: The T2FLFNN parameters are coded into a vector. The parameters include uncertainty mean $m_{i j}$, standard deviation $\sigma_{i j}$, and link weight $\omega_{k j}$. Then the target vector $X_{D, G}^{i}$ is randomly initialized in the solution space. The mathematical model is expressed as

$$
X_{D, G}^{i}=\left[x_{1, G}^{i}, x_{2, G}^{i}, \ldots, x_{D, G}^{i}\right]
$$

\section{LDGDE Algorithm Pseudocode}

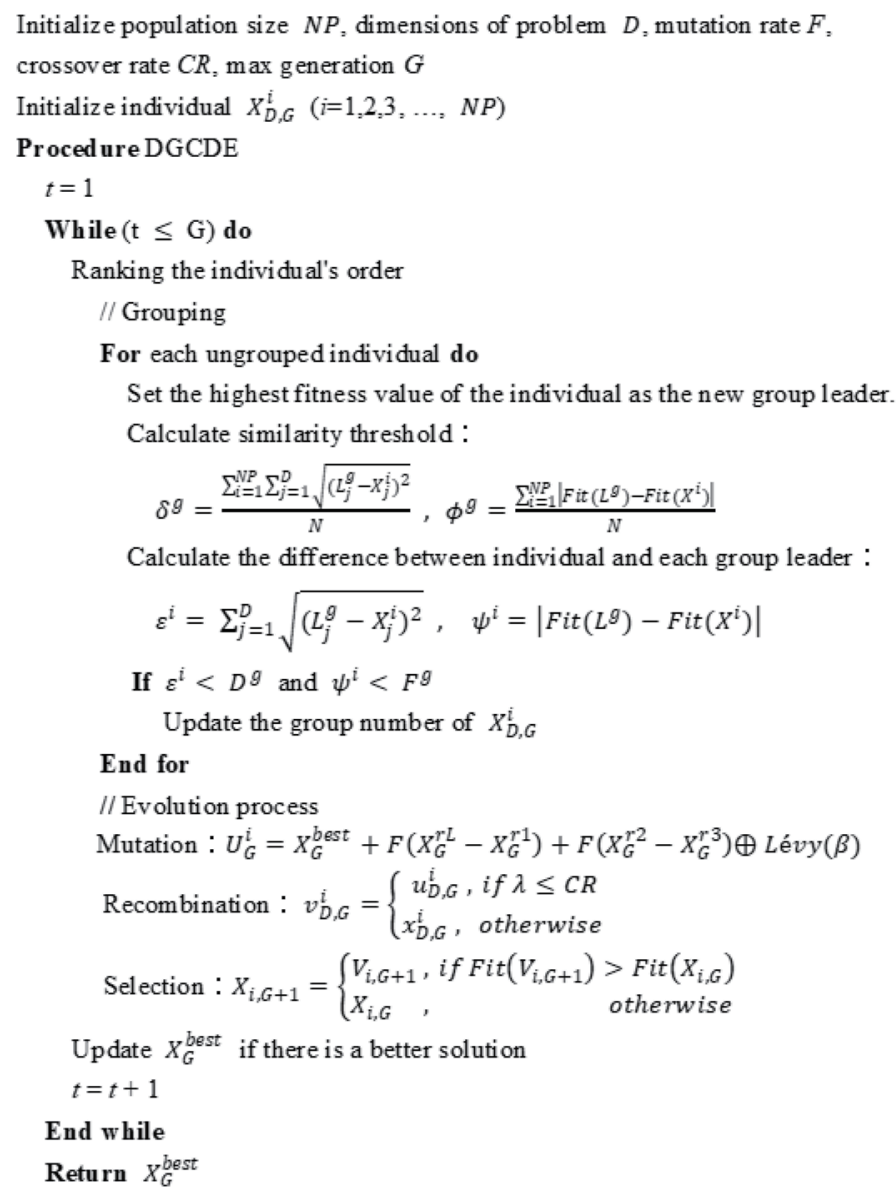

Fig. 6. Pseudocode of T2FLFNN. 
Here, $i=1,2, \ldots, N P$. NP represents the size of the population, $G$ is the current generation number, and $D$ is the dimension.

Vector grouping: The group number of the initial state is 0 . The vectors are sorted in descending order of fitness values. The thresholds of fitness and distance are calculated as the average difference in the distance and the average difference in the fitness between the ungrouped vector and the group leader in group number 0 , respectively.

$$
\begin{gathered}
\delta^{g}=\sum_{i=1}^{N P} \sum_{j=1}^{D} \sqrt{\left(L_{j}^{g}-X_{j}^{i}\right)^{2}} \\
\varphi^{g}=\sum_{i=1}^{N P}\left|\operatorname{Fit}\left(L^{g}\right)-\operatorname{Fit}\left(X^{i}\right)\right| \\
D^{g}=\frac{\delta^{g}}{N} \\
F^{g}=\frac{\varphi^{g}}{N}
\end{gathered}
$$

Here, $\delta^{g}$ and $\varphi^{g}$ are the differences in the distance and fitness between the ungrouped vectors and the group leader, $D^{g}$ and $F^{g}$ represent threshold values of fitness and distance, $L_{j}^{g}$ is the jth dimension of the gth group leader, and $N$ is the total number of ungrouped vectors, respectively. To group the vectors, the difference in the distance value $\left(\varepsilon^{\dot{ }}\right)$ and the difference in the fitness value $\psi^{i}$ between the ungrouped vectors and the leader vectors are calculated as

$$
\begin{gathered}
\varepsilon^{i}=\sum_{j=1}^{D} \sqrt{\left(L_{j}^{g}-X_{j}^{i}\right)^{2}}, \\
\psi^{i}=\left|\operatorname{Fit}\left(L^{g}\right)-\operatorname{Fit}\left(X^{i}\right)\right| .
\end{gathered}
$$

The group number of the vector is updated to $g$ if $\varepsilon^{i}>D^{g}$ and $\psi^{i}<F^{g}$.

Mutation: We propose a new mutation method that introduces the Lévy flight strategy and refers to the group leader position to enhance the efficiency of the traditional DE algorithm. The mutation formula can be expressed as

$$
\begin{gathered}
U_{G}^{i}=X_{G}^{b e s t}+F\left(X_{G}^{r L}-X_{G}^{r 1}\right)+F\left(X_{G}^{r 2}-X_{G}^{r 3}\right) \oplus \operatorname{Lévy}(\beta), \\
\operatorname{Lévy}(\beta)=\frac{\mu}{|\nu|^{1 / \beta}},
\end{gathered}
$$




$$
\begin{gathered}
\mu=N\left(0, \sigma_{\mu}^{2}\right), v=N\left(0, \sigma_{v}^{2}\right), \\
\sigma_{\mu}=\left[\frac{\Gamma(1+\beta) \sin (\pi \beta / 2)}{\Gamma\left(1+\frac{\beta}{2}\right) \beta 2^{(\beta-1) / 2}}\right]^{1 / \beta}, \sigma_{v}^{2}=1,
\end{gathered}
$$

where $U_{G}^{i}=\left[u_{1, G}^{i}, u_{2, G}^{i}, \ldots, u_{D, G}^{i}\right]$ is the mutant vector, $X_{G}^{\text {best }}$ is the best fitness vector, $F$ is the mutation weight factor, $X_{G}^{r L}$ is a random leader, $\beta=1.5$ is the Lévy flight exponent, and $\mu$ and $v$ represent Gaussian normal distributions with a zero mean and variances of $\sigma_{\mu}^{2}$ and $\sigma_{v}^{2}$, respectively.

Recombination: To generate a new trial vector $V_{G}^{i}=\left[v_{1, G}^{i}, v_{2, G}^{i}, \ldots, v_{D, G}^{i}\right]$, the following formula is used to cross the mutation vector with the target vector:

$$
v_{D, G}^{i}= \begin{cases}u_{D, G}^{i}, & \text { if } \lambda \leq C R, \\ x_{D, G}^{i}, & \text { otherwise, }\end{cases}
$$

where $\lambda \in(0,1)$ represents random values of each dimension and $C R$ is the crossover rate.

Selection: The next generation of target vectors is selected on the basis of the evaluated fitness value. The target vector will remain if the fitness value of the trial vector is worse than that of the current target vector. This is described as

$$
X_{i, G+1}= \begin{cases}V_{i, G+1}, & \text { if } \operatorname{Fit}\left(V_{i, G+1}\right)>\operatorname{Fit}\left(X_{i, G}\right), \\ X_{i, G}, & \text { otherwise. }\end{cases}
$$

\subsection{Optimization of grinding parameters}

In this study, the IGAS combined with the T2FLFNN prediction model and PSO algorithm is proposed to assist the user in grinding parameter selection. The mathematical model is defined as

$$
\begin{gathered}
V_{i}(n+1)=\omega V_{i}(n)+C_{1} \varphi_{1}\left(P_{b e s t}-X_{i}(n)\right)+C_{2} \varphi_{2}\left(G_{\text {best }}-X_{i}(n)\right), \\
X_{i}(n+1)=X_{i}(n)+V_{i}(n+1) .
\end{gathered}
$$

Here, $V_{i}(n)$ represents the velocity for particle $X_{i}(n), \omega$ represents the inertia weight, $G_{b e s t}$ is the best particle among the particles, $P_{\text {best }}$ is the best particle among the current particles, $C_{1}$ and $C_{2}$ 
are the cognitive parameter and social parameter, respectively, and $\varphi_{1}$ and $\varphi_{2}$ are randomly set between 0 and 1 . The fitness function $F(\cdot)$ is used to evaluate whether the grinding parameters satisfy the quality requirements of the user, where

$$
F(\cdot)=\frac{1}{1+\left(\left|T_{a}-M_{a}\right|\right)} .
$$

Here, $M_{a}$ represents the surface roughness obtained from the prediction model and $T_{a}$ represents the grinding target $R a$ set by the operator.

\section{Experimental Results}

The full factorial design method is employed to collect the experimental data in this section. To verify the Ra prediction model effectively, the proposed T2FLFNN is compared with the $\mathrm{BPNN}^{(24)}$ and FNN. In addition, PSO is used for grinding parameter optimization, and different target $\mathrm{Ra}$ values are used to generate grinding parameters in the actual grinding process for verification.

\subsection{Data collection}

The experimental data are collected by the full factorial design method. Table 6 presents the grinding parameters. The grinding parameters are the axial depth of cut, cutting speed, feed rate, ultrasonic power, and radial depth of cut. A total of 2200 data are collected to establish the prediction. The training data and testing data are divided in the ratio of 80 to $20 \%$.

\subsection{Ra prediction results}

The proposed T2FLFNN is compared with the BPNN and FNN to verify the effectiveness of the prediction model. The dataset is split into 1760 training data and 440 testing data for building the prediction model. Each model is trained by 3000 iterations, then its effectiveness is evaluated using the mean absolute percentage error (MAPE), defined as

$$
M A P E=\frac{100 \%}{n} \sum_{i=1}^{n}\left|\frac{\hat{y}_{i}-y_{i}}{y_{i}}\right|,
$$

Table 6

Parameters of grinding experiment.

\begin{tabular}{lc}
\hline Axial depth of cut $(\mathrm{mm})$ & $0.004,0.02$ \\
\hline Cutting speed $(\mathrm{mm} / \mathrm{min})$ & $75,90,105,120,135,150,165,180,195,210,225$ \\
\hline Feed rate $(\mathrm{mm} / \mathrm{min})$ & $70,80,90,100,110,120,130,140,150,160,170$, \\
& $180,190,200,210,220,230,240,250,260$ \\
\hline Ultrasonic power $(\%)$ & $0,10,30,60,100$ \\
\hline Radial depth of cut $(\mathrm{mm})$ & 5.5 \\
\hline
\end{tabular}


where $\hat{y}_{i}$ is the actual measured $R a$ value, $n$ is the number of data, and $y_{i}$ is the predicted value. The performance of each prediction model is shown in Fig. 7 and Table 7. The BPNN has the highest MAPE (7.89\%) among the prediction models. The FNN combines fuzzy logic and a neural network; therefore, the MAPE (6.07\%) is better than that of the BPNN. Compared with

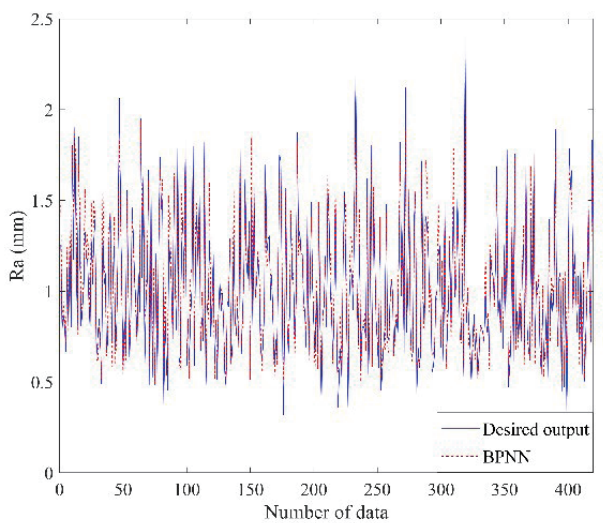

(a)

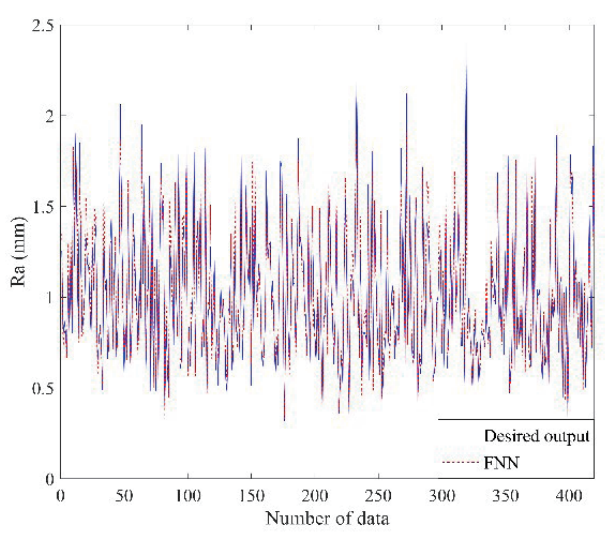

(c)

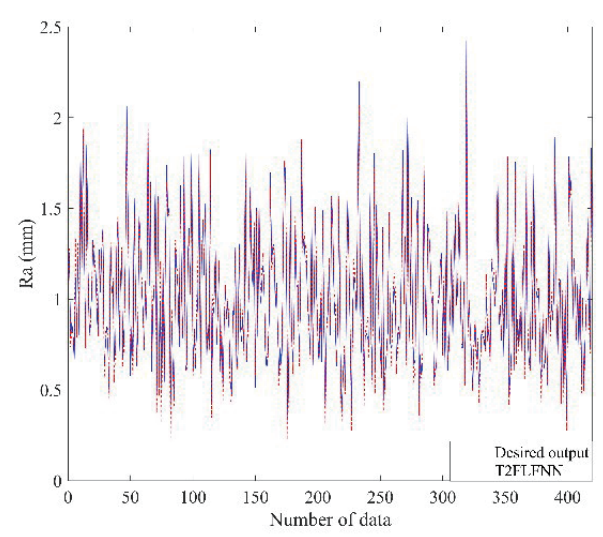

(e)

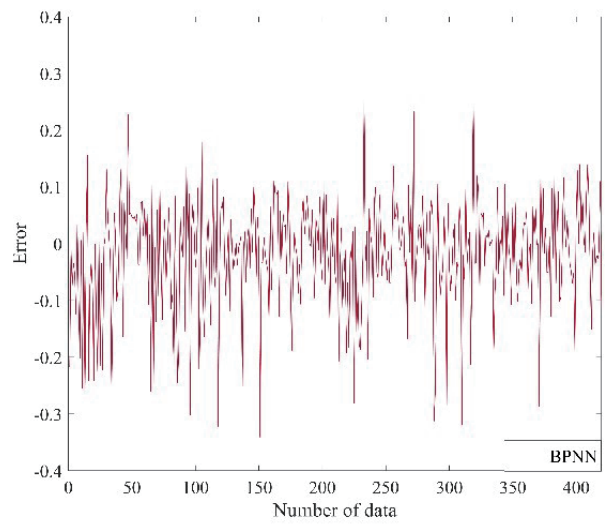

(b)

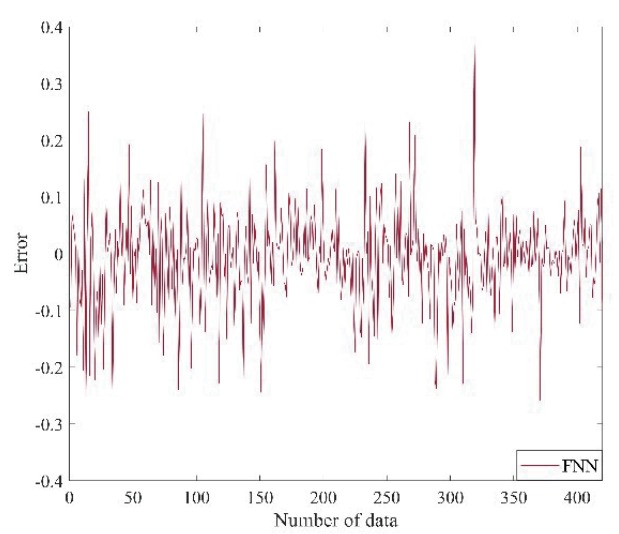

(d)

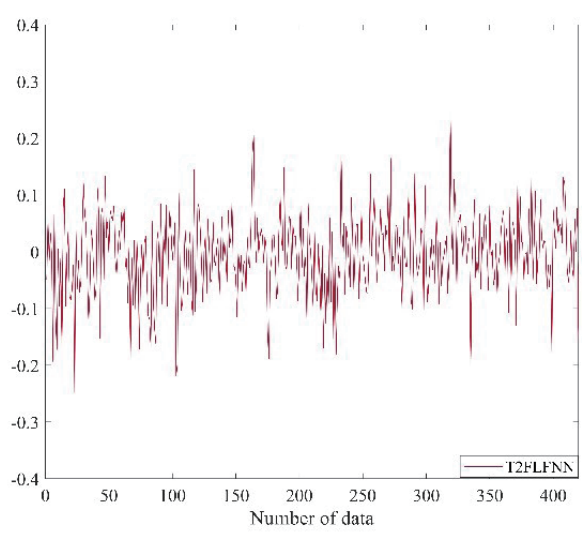

(f)

Fig. 7. (Color online) Prediction results of various models: (a) BPNN, (b) prediction error using BPNN, (c) FNN, (d) prediction error using FNN, (e) T2FLFNN, and (f) prediction error using T2FLFNN. 
Table 7

Prediction performance of each model.

\begin{tabular}{lc}
\hline Model & MAPE (\%) \\
\hline BPNN & 7.89 \\
FNN & 6.07 \\
T2FLFNN & 5.04 \\
\hline
\end{tabular}

Table 8

Initial parameters of PSO.

\begin{tabular}{cccc}
\hline Particles & $\omega$ & $C_{1}, C_{2}$ & Generations \\
\hline 40 & 0.5 & 2 & 200 \\
\hline
\end{tabular}

the BPNN ${ }^{(25)}$ and FNN, the proposed T2FLFNN with the LDGDE parameter learning method has a lower MAPE (5.04\%). This indicates that the type 2 fuzzy set adopted by the proposed T2FLFNN has the best performance. In addition, the accuracy of the predictive model is crucial for optimizing the grinding processing parameters for performance. If the accuracy of the prediction model is insufficient, the parameters optimized by the evolutionary algorithm will not be able to obtain the expected results. The results showed that the T2FLFNN is sufficiently accurate to establish an Ra prediction model.

\subsection{Grinding parameter optimization results}

Different combinations of grinding parameters will produce different Ra. In this subsection, PSO is used to solve the parameter optimization problem. The initial parameters of PSO are shown in Table 8. The parameters of PSO are the number of generations, the acceleration constants $C_{1}$ and $C_{2}$, the single inertia weight $\omega$, and the number of particles. In this experiment, 11 different target values of $\mathrm{Ra}$ are adopted to generate grinding processing parameters. The generated parameters are used in the MV184C CNC machine for grinding processing to verify the performance. The experimental results are shown in Fig. 8 and Table 9. As shown in Table 9, the 11 sets of parameters generated by the IGAS have an MAPE of $1.62 \%$. This indicates that the proposed IGAS can assist users in selecting grinding parameters in line with their processing quality requirements.

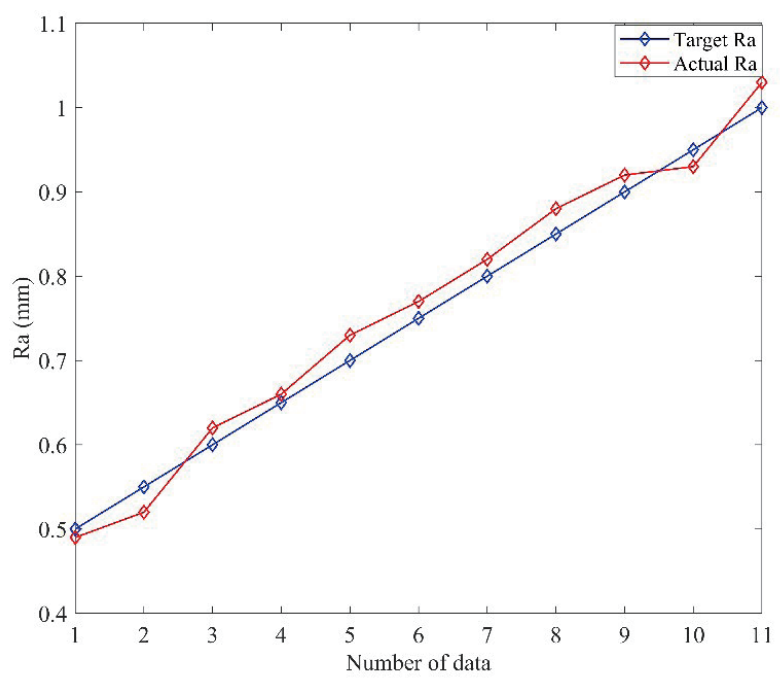

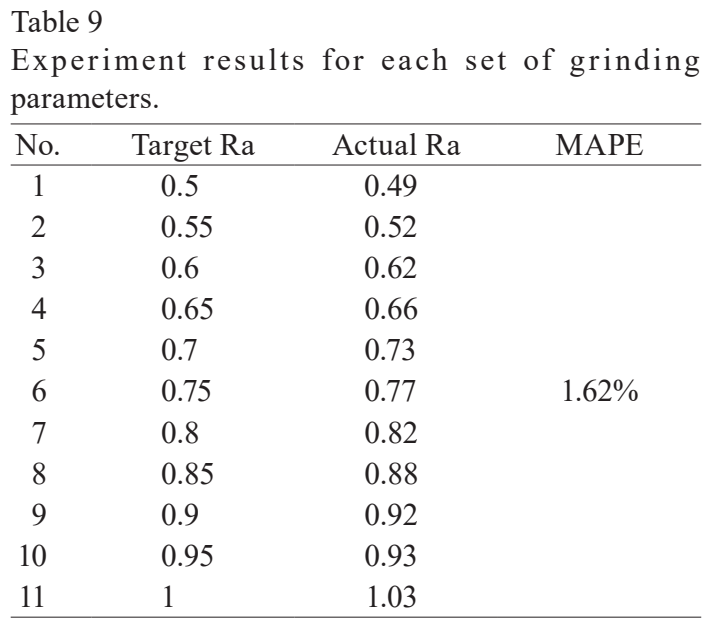

Fig. 8. (Color online) Experimental results of grinding processing. 


\section{Conclusions}

We introduced a novel IGAS to assist the user in the grinding process. The proposed IGAS uses a T2FLFNN to establish a prediction model, then PSO is used to obtain the optimized grinding parameters. To improve the accuracy of the T2FLFNN, LDGDE, which includes the Lévy flight and dynamic group mechanism, is developed to update the network parameters. The MAPE experimentally obtained using the proposed T2FNN is $6.07 \%$ and is superior to the values obtained with the BPNN and FNN. In addition, 11 different target values of Ra are adopted to generate grinding processing parameters. The generated parameters are used in an MV184C CNC machine for grinding processing to verify the performance of the proposed IGAS. The experimental results of practical machining show that the MAPE using the IGAS is as low as $1.62 \%$. Therefore, the proposed IGAS can provide suitable grinding parameters according to the requirements of users.

\section{Acknowledgments}

The authors would like to thank the Ministry of Science and Technology of the Republic of China, Taiwan, for financially supporting this research under Contract Nos. MOST 109-2218-E005-002 and MOST 109-2634-F-009-031.

\section{References}

1 I. Laird, X. Yuan, J. Scoltock, and A. J. Forsyth: IEEE Trans. Power Electron. 33 (2018) 2913. https://doi. org/10.1109/TPEL.2017.2705805

2 K. Gould, S. Q. Cai, C. Neft, and A. Bhunia: IEEE Trans. Power Electron. 30 (2015) 2975. https://doi. org/10.1109/TPEL.2014.2331562

3 S. Singh, K. Rathi, and K. Pal: J. Alloys Compd. 740 (2018) 436. https://doi.org/10.1016/j.jallcom.2017.12.069

4 Y. Liu, Z. Liu, X. Wang, and T. Huang: Int. J. Adv. Manuf. Technol. 107 (2020) 425. https://doi.org/10.1007/ s00170-020-05060-Z

5 D. Wang, S. Lu, D. Xu, and Y. Zhang: Materials 13 (2020) 1918. https://doi.org/10.3390\%2Fma13081918

6 J. Chen, W. Ming, Q. An, and M. Chen: Ceram. Int. 46 (2020) 15122. https://doi.org/10.1016/j. ceramint.2020.03.047

7 D. Kong, J. Zhu, C. Duan, L. Lu, and D. Chen: Mech. Syst. Sig. Process. 142 (2020) 106770. https://doi. org/10.1016/j.ymssp.2020.106770

8 A. Sudianto, Z. Jamaludin, and A. A. A. Rahman: J. Phys.: Conf. Ser. 1201 (2019) 1742. https://iopscience.iop. org/article/10.1088/1742-6596/1201/1/012008

9 R. R. Srikant, P. V. Krishna, and N. D. Rao: Proc. Inst. Mech. Eng. 225 (2011) 1009. https://doi. org//10.1177/0954405410395854

10 S. Cheri, J. Wang, and T. Gu: Proc. 2018 37th Chinese Control Conf. (IEEE, 2018) 8330.

11 A. Sharifian, M. J. Ghadi, S. Ghavidel, L. Li, and J. Zhang: Renewable Energy 120 (2018) 220. https://doi. org/10.1016/j.renene.2017.12.023

12 C. M. Lin, T. L. Le, and T. T. Huynh: Neurocomputing 275 (2018) 2239. https://doi.org/10.1016/j. neucom.2017.11.009

13 Y. Lin, S. Liao, J. Chang, and C. Lin: IEEE Trans. Neural Networks Learn. Syst. 25 (2014) 959. https://doi. org/10.1109/TNNLS.2013.2284603

14 S. A. Zaheer, S. Choi, C. Jung, and J. Kim: IEEE/ASME Trans. Mechatron. 20 (2015) 3182. https://doi. org/10.1109/TMECH.2015.2411853

15 N. Leema, H. K.Nehemiah, and A. Kannan: Appl. Soft Comput. 49 (2016) 834. https://doi.org/10.1016/j. asoc.2016.08.001 
16 T. Eltaeib and A. Mahmood: Appl. Sci. 8 (2018) 1945. https://doi.org/10.3390/app8101945

17 C. J. Lin, J. Y. Jhang, S. H. Chen, and K. Y. Young: IEEE Access 8 (2020) 122626. https://doi.org/10.1109/ ACCESS.2020.3006849

18 J. C. Bansal: Evolutionary and Swarm Intelligence Algorithms, P. K. Singh and N. R. Pal, Eds. (Springer International Publishing, Cham, 2019) Chap. 2.

19 H. Rao, X. Shi, A. K. Rodrigue, J. Feng, Y. Xia, M. Elhoseny, X. Yuan, and L. Gu: Appl. Soft Comput. 7 (2019) 634. https://doi.org/10.1016/j.asoc.2018.10.036

20 A. H. Hamamoto, L. F. Carvalho, L. D. H. Sampaio, T. Abrão, and M. L. Proença: Expert Syst. Appl. 92 (2018) 390. https://doi.org/10.1016/j.eswa.2017.09.013

21 S. Mirjalili and A. Lewis: Adv. Eng. Software 95 (2016) 51. https://doi.org/10.1016/j.advengsoft.2016.01.008

22 Product BT Series: Hantop Intelligence Tech (2018).

23 MV184 Specification: QUASER (2019).

24 NewView ${ }^{\text {TM }}$ Product Manuals: ZYGO (2017).

25 N. B. Fredj and R. Amamou: Int. J. Adv. Manuf. Technol. 31 (2006) 24. https://doi.org/10.1007/s00170-0050169-8

\section{About the Authors}

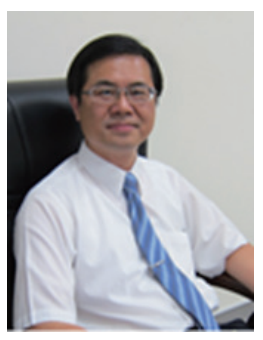

Cheng-Jian Lin received his B.S. degree in electrical engineering from Ta Tung Institute of Technology, Taipei, Taiwan, R.O.C., in 1986 and his M.S. and $\mathrm{Ph}$.D. degrees in electrical and control engineering from National Chiao Tung University, Taiwan, R.O.C., in 1991 and 1996, respectively. Currently, he is a chair professor of the Computer Science and Information Engineering Department, National Chin-Yi University of Technology, Taichung, Taiwan, R.O.C., and dean of Intelligence College, National Taichung University of Science and Technology, Taichung, Taiwan, R.O.C. His current research interests are machine learning, pattern recognition, intelligent control, image processing, intelligent manufacturing, and evolutionary robots.

(cjlin@ncut.edu.tw)

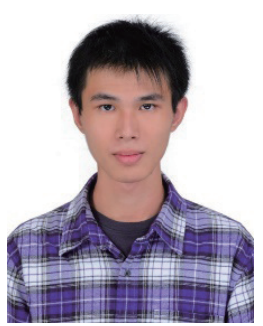

Jyun-Yu Jhang received his B.S. and M.S. degrees from the Department of Computer Science and Information Engineering, National Chin-Yi University of Technology, Taichung, Taiwan, in 2015. He is currently pursuing his Ph.D. degree with the Institute of Electrical and Control Engineering, National Yang Ming Chiao Tung University, Hsinchu, Taiwan. His current research interests include fuzzy logic theory, type-2 neural fuzzy systems, evolutionary computation, machine learning, computer vision, and applications.

(o800920@yahoo.com.tw)

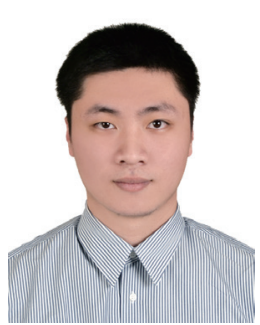

Shou-Zheng Huang received his B.S. and M.S. degrees from the Department of Mechanical Engineering, National Chin-Yi University of Technology, Taichung, Taiwan in 2017. He is currently pursuing his Ph.D. degree with the Department of Mechanical Engineering, National Chin-Yi University of Technology, Taichung, Taiwan. His current research interests include precision machining, grinding, polishing processes, and numerical simulation. (a0980787078@gmail.com) 
Ming-Yi Tsai received his Ph.D. degree in mechanical engineering from National Taiwan University, Taiwan, in 2006. He is currently a professor of mechanical engineering of National Chin-Yi University of Technology. His current research interests include precision machining, grinding, polishing processes, and numerical simulation. (mytsai@ncut.edu.tw) 